\title{
Dialnet Métricas como herramienta de evaluación bibliométrica: aportes al análisis de la actividad científica en Ciencias Sociales y Humanidades
}

\section{Dialnet Metrics as a bibliometric evaluation tool: contributions to the analysis of the scientific activity in Social Sciences and Humanities}

\author{
Orlando Gregorio-Chaviano; Rafael Repiso; Antonio Calderón-Rehecho; \\ Joaquín León-Marín; Evaristo Jiménez-Contreras
}

Cómo citar este artículo:

Gregorio-Chaviano, Orlando; Repiso, Rafael; Calderón-Rehecho, Antonio; León-Marín, Joaquín; JiménezContreras, Evaristo (2021). "Dialnet Métricas como herramienta de evaluación bibliométrica: aportes al análisis de la actividad científica en Ciencias Sociales y Humanidades". Profesional de la información, v. 30, n. 3, e300318.

https://doi.org/10.3145/epi.2021.may.18

Artículo recibido el 12-02-2021

Aceptación definitiva: 15-05-2021
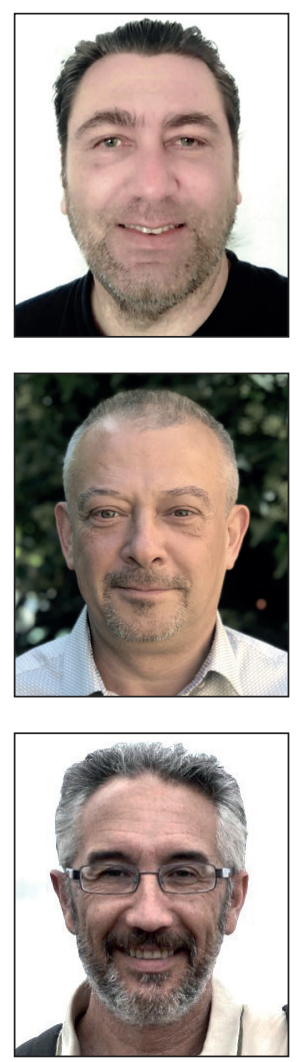

Orlando Gregorio-Chaviano $\square$ https://orcid.org/0000-0002-3064-8639

Pontificia Universidad Javeriana Facultad de Comunicación y Lenguaje Depto. de Ciencia de la Información

Transversal 4, n. 42-00, Piso 6. Edif. 67

Bogotá, Colombia

ogregorio@javeriana.edu.co

Antonio Calderón-Rehecho https://orcid.org/0000-0002-9948-2825

Universidad Complutense de Madrid Biblioteca. Edificio Multiusos

Ciudad Universitaria

Profesor Aranguren, s/n.

28040 Madrid, España

acaldero@ucm.es

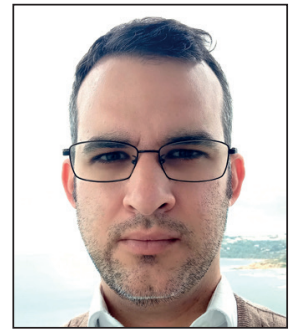

Rafael Repiso

https://orcid.org/0000-0002-2803-7505

Universidad Internacional de La Rioja

Av. de la Paz, 137

26006 Logroño, España

rafael.repiso@gmail.com

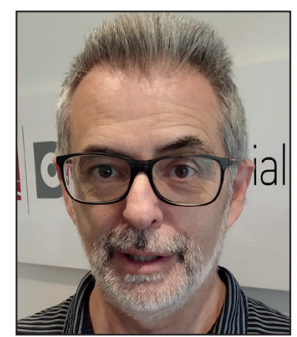

Joaquín León-Marín

https://orcid.org/0000-0003-2906-9864

Fundación Dialnet

Universidad de La Rioja

Barriocepo, 10.

26001 Logroño, España

joaquin.leon@unirioja.es

\section{Resumen}

Dentro del panorama actual de evaluación de la ciencia, las limitaciones de los índices de citación para estudiar las Ciencias Sociales y Humanidades han sido motivo de amplio debate. Para resolver esta situación, se han creado distintos productos para ser usados en contextos nacionales, dado que cubren ciertos aspectos no presentes en los índices de carácter más internacional. Como ejemplo se encuentran los de la familia In-RECS, donde se define un indicador similar al factor de impacto de Eugene Garfield, pero su aporte radica en la capacidad de evaluar la investigación en España mediante la obtención de indicadores de citas. Es por ello por lo que en este trabajo se expone la necesidad de crear nuevos productos para la evaluación de la investigación en general, pero particularizando en las Ciencias Sociales y Humanidades. Se muestra el 
contexto en el que surgen y se desarrollan las distintas alternativas de evaluación de revistas existentes y se presenta Dialnet Métricas. Este es un índice de citación realizado por la Fundación Dialnet en colaboración con el Grupo EC3 y decenas de universidades españolas. A partir del análisis de las referencias citadas de revistas fuente de distintos campos temáticos, Dialnet Métricas proporciona indicadores para evaluar el impacto de la investigación a varios niveles. Este producto bibliométrico posibilita el análisis contextualizado a nivel micro (investigadores), meso (revistas) y macro (áreas y universidades). Por último, se describen cuantitativamente los contenidos, volúmenes de datos y estructura de este índice de citas.

\title{
Palabras clave
}

Dialnet Métricas; Índices de citas; Indicadores; Bibliometría; Evaluación científica; Clasificaciones; Rankings; Colaboración; Bibliotecas universitarias; Información científica; Evaluación; Calidad; Impacto.

\begin{abstract}
Within the current panorama of science evaluation, the limitations of citation indexes to study the social sciences and humanities have been the subject of wide debate. To resolve this situation, different products have been created for use in national contexts, since they cover certain aspects not contained in more international indices. An example is the In-RECS family, where an indicator such as the impact factor of Eugene Garfield is defined, but its contribution lies in the ability to evaluate research in Spain by obtaining citation indicators. This paper thus highlights the need to create new products for research evaluation in general, but particularly in the social sciences and humanities. The context in which different alternatives arise and are developed to evaluate existing journals is presented, along with Dialnet Metrics, a citation index developed by the Dialnet Foundation in collaboration with the EC3 Group and dozens of Spanish universities. Based on an analysis of the citations of source journals from different subject areas, Dialnet Metrics provides indicators to evaluate the research impact at different levels. This bibliometric product enables contextualized analysis at the micro (researchers), meso (journals), and macro (areas and universities) levels. Finally, the content, data volumes, and structure of this citation index are described quantitatively.
\end{abstract}

\section{Keywords}

Dialnet Metrics; Citation indexes; Bibliometrics; Indicators; Scientific evaluation; Rankings; Journals; Institutions; Researchers; Classifications; Collaboration; University libraries; Scientific information; Scholarly information; Academic information; Evaluation; Quality; Impact.

\section{Contexto de aparición de alternativas para la medición de la ciencia}

Las revistas científicas son indispensables como registro y certificación del conocimiento generado por los investigadores, además de ser instrumentos clave para conocer la historia de las disciplinas científicas, de los procesos de institucionalización de las mismas y principal medio de comunicación científica entre todos los canales formales e informales (Delgado-López-Cózar, 2017). Como difusoras del conocimiento son un medio principal y uno de los más habitualmente empleados para la evaluación de la investigación (Alperin; Rozemblum, 2017).

El interés por las revistas, no sólo como medios de difusión y método de análisis de las comunidades, es antiguo y cubre un espectro amplio de enfoques y realidades. Elementos relacionados con el conteo de citas, la gestión de las colecciones en bibliotecas, la toma de decisiones en política científica, así como su evaluación de cara al ingreso en bases de datos y repertorios bibliográficos (Delgado-López-Cózar, 2017), han sido motivos por los que se les ha evaluado desde su origen, al igual que los autores que en ellas publican.

Aunque la evaluación de la actividad investigadora es una necesidad sentida desde antiguo, los métodos actuales se consolidaron en gran medida con la creación del Institute for Scientific Information (ISI) en 1956 y la propuesta de Eugene Garfield del indicador denominado factor de impacto (Garfield, 1999; 2003). Este permitió conocer la relevancia de las publicaciones a partir del registro de la producción y el conteo de citas e impulsó las políticas de selección de publicaciones de las bases de datos que se mantienen aún vigentes en Web of Science y en otros sistemas análogos. Los resultados de la evaluación de revistas hacen posible entender las prácticas de comunicación y publicación de las disciplinas, la forma en que se distribuye el éxito y las recompensas, además de favorecer el control del conocimiento científico generado de acuerdo a las tendencias de cada área (Delgado-López-Cózar, 2017).

En este escenario, la existencia de bases de datos y sistemas encargados de la evaluación y clasificación de revistas, como la diversidad de indicadores bibliométricos (Torres-Salinas; Jiménez-Contreras, 2010) y el debate sobre la pertinencia de las fuentes y su representatividad estadística (Chavarro; Ràfols; Tang, 2018; López-López, 2018), son elementos esenciales que han traído constantes cambios y mejoras de los productos existentes y la aparición de otros nuevos.

Por otro lado y en el caso de las Ciencias Sociales y las Humanidades, a los anteriores se suman otros aspectos como la orientación nacional de la investigación, el predominio de canales de comunicación distintos al artículo científico, la tendencia a publicar más en revistas nacionales y los hábitos de producción y consumo que difieren de los de otras áreas (Giménez-Toledo, 2018), con la consiguiente pérdida de eficacia de los indicadores bibliométricos. Todas son cuestiones incorporadas en los últimos años al mencionado debate y han impulsado la creación de nuevos productos de evaluación surgidos como respuesta al monopolio de los sistemas tradicionales de evaluación (Torres-Salinas; Repiso, 2016). 
En España, el grupo de recursos de la llamada familia In-RECX (In-RECS, In-RECJ e In-RECH) representó una importante herramienta que como índice de impacto se encargó del recuento de las referencias bibliográficas para determinar la influencia, relevancia e impacto de las revistas españolas, de los autores y de las instituciones de Ciencias Sociales y Derecho (Delgado-López-Cózar et al., 2005), usados no sólo por las agencias nacionales para la evaluación de las revistas y la investigación, sino por otras bases de datos en el proceso de selección de revistas.

En su momento ofrecieron recursos a las agencias de evaluación, revistas e investigadores, para la gestión de la actividad científica, avanzando notablemente tanto en la dirección de poner a disposición de la comunidad información elaborada sobre las revistas españolas, hasta entonces carentes de indicadores, la elaboración de nuevos indicadores y una profundización considerable en los niveles de análisis ofrecidos. Sin embargo, los costos económicos, la ausencia de fondos y las dificultades técnicas relacionadas con la captura y procesamiento de información, han hecho difícil la creación o el mantenimiento de este tipo de productos alternos sin fuentes económicas estables.

Aunque no basados en la contabilización de citas, otros como la Clasificación Integrada de Revistas (CIRC) del Grupo EC3; Difusión y Calidad Editorial de las Revistas Españolas de Humanidades y Ciencias Sociales y Jurídicas (DICE); Revistas Españolas de Ciencias Sociales y Humanidades (RESH) (Giménez-Toledo, 2015; Torres-Salinas et al., 2010; Torres-Salinas; Repiso, 2016), son instrumentos de valoración del comportamiento a partir del cumplimiento de criterios de calidad y mecanismos importantes para la evaluación.

Cabe señalar que muchas de las iniciativas han desaparecido igualmente por problemas de financiación (ejemplo de ello son los recursos de la llamada familia In-RECX). Por último, para concluir la enumeración de las españolas y gozando además aparentemente de buena salud hay que mencionar la Matriz de Información para el Análisis de Revistas (MIAR) cuyo alcance es mucho mayor, siendo de hecho un repertorio de revistas de todas las especialidades valoradas en base a su difusión y visibilidad internacional (Rodríguez-Gairín; Somoza-Fernández; Urbano, 2011).

En la misma línea se encuentran productos elaborados en el contexto latinoamericano como:

- Sistema Brasileño de Evaluación de Revistas (Qualis) (Martínez-Ávila, 2019);

- UCRindex de Costa Rica (Córdoba-González; Murillo-Goussen; Polanco-Cortés, 2017);

- Índice Bibliográfico Nacional Publindex de Colombia (Charum, 2004);

- Índice Mexicano de Evaluación de Revistas (Vasen; Lujano-Vilchis, 2017);

- Redib (Abejón-Peña, 2018).

Todos ellos contribuyen a la visibilidad de las revistas nacionales y regionales.

En su mayoría los anteriores se centran más en el cumplimiento de criterios como condición de inclusión y posicionamiento en sus categorías y no tanto en el cálculo de citas. Esto es una tendencia relacionada con la insuficiencia de productos alternativos a WoS y Scopus para la evaluación de revistas y de la actividad científica, situación más marcada en el campo de las Ciencias Sociales y Humanidades y cuando se desciende a los estudios regionales, razón que justifica la creación de nuevas propuestas.

A lo anterior debemos añadir el liderazgo que en Latinoamérica siguen ejerciendo sistemas como Redalyc (Aguado-López et al., 2008) y SciELO (Packer et al., 2006), que buscan fortalecer la visibilidad de la producción científica regional y ser de utilidad como instrumentos de evaluación de las comunidades. En todo caso es significativo el apoyo que brindan a la consolidación de las revistas regionales y a su modelo de comunicación científica para el fortalecimiento de la producción latinoamericana.

En la tabla 1 se presenta un resumen de las características y prestaciones más relevantes de los productos mencionados hasta ahora en relación con su capacidad de evaluar revistas (no se incluye CIRC ni MIAR pues no hacen evaluación en sentido estricto).

Tabla 1. Descripción de productos que evalúan revistas

\begin{tabular}{|c|c|c|c|c|c|c|c|}
\hline & $\begin{array}{c}\text { Dialnet } \\
\text { Métricas }\end{array}$ & $\begin{array}{l}\text { Web of } \\
\text { Science }\end{array}$ & Scopus & In-RECX & Redib & $\begin{array}{c}\text { Ranking } \\
\text { Fecyt }\end{array}$ & SJR \\
\hline N. de revistas & 2.472 & $34.586^{*}$ & 38.589 & 1.650 & 3.758 & 409 & 26.201 \\
\hline Áreas & CSS/ Hum. & Todas & Todas & CSS/ Hum. & Todas & Todas & Todas \\
\hline Geografía & España & Mundial & Mundial & España & Iberoamérica & España & Mundial \\
\hline Período & 2016- & 1979- & 1999- & 1996-2011 & 2017- & 2018- & 1999- \\
\hline Transparencia & Buena & Buena & Buena & Buena & Dudosa & Opaca & Opaca \\
\hline Fuente de datos & Propia & Propia & Propia & Propia & Ajena & Ajena & Ajena \\
\hline Métrica de artículos & Sí & Sí & Sí & Sí & No & No & No \\
\hline Acceso & Gratuito & Pago & Pago & Gratuito & Pago & Gratuito & Gratuito \\
\hline Responsable & $\begin{array}{l}\text { Proyecto } \\
\text { colaborativo }\end{array}$ & $\begin{array}{l}\text { Empresa } \\
\text { privada }\end{array}$ & $\begin{array}{l}\text { Empresa } \\
\text { privada }\end{array}$ & $\begin{array}{l}\text { Grupo de } \\
\text { investigación }\end{array}$ & $\begin{array}{l}\text { Instituto de } \\
\text { investigación }\end{array}$ & Fundación & $\begin{array}{l}\text { Empresa } \\
\text { privada }\end{array}$ \\
\hline
\end{tabular}

*Sólo se tienen en cuenta las revistas Core de Web of Science en la Master List.

**Al referirnos a opacos o dudosos se debe a que las mencionadas iniciativas no explican los criterios. 
En este contexto, ante la insuficiencia de los medios disponibles, por abandono de algunos y por falta de información bibliométrica de otros, surge Dialnet Métricas $(D M)$, sistema que a partir del análisis de las referencias bibliográficas citadas de las publicaciones existentes en Dialnet (Dialnet, 2020), provee un conjunto de indicadores bibliométricos para conocer la relevancia de la producción en los distintos agregados científicos y llega para dar respuesta a la perenne necesidad de evaluación de las Ciencias Sociales y las Humanidades, siempre atendida de forma irregular por las dificultades ya mencionadas.

De esta forma, la posibilidad de obtener información sobre el impacto y demás indicadores de las revistas españolas (o sobre los autores e instituciones), no incluidas en las bases de datos de mayor relevancia representa una importante alternativa. Además, el conjunto de revistas ahora analizadas en Dialnet Métricas supone un porcentaje muy importante, a veces mayoritario, de las utilizadas por los investigadores en estas áreas.

En el presente trabajo se describen la estructura, proceso de selección de revistas, volumen de datos, así como la metodología de elaboración y los indicadores que suministra.

\section{Descripción del índice y criterios de inclusión de revistas}

Como en su momento propuso Garfield (1955) y más tarde todos los índices construidos con criterios bibliométricos, la base de datos Dialnet Métricas (DM) se ha diseñado en dos fases.

En primer lugar se partió de la identificación de las poblaciones de revistas de cada especialidad que permitiera realizar una selección de la muestra con mayor representatividad y posteriormente registrar las referencias citadas de sus artículos usando un programa que enlaza las referencias de los artículos de las revistas fuente con trabajos de la población objeto (revistas nacionales).

Para la identificación de las poblaciones de revistas de cada campo se utilizó el repertorio Latindex con añadidos de Dialnet, seleccionando en cada especialidad las españolas académicas vivas, que estuvieran o no incluidas en el catálogo y declararan emplear la evaluación por pares. Aunque estos criterios, seleccionados y aplicados desde una perspectiva operativa, basados en la selección de revistas radicadas en España, no se consideraron discriminantes por sí mismos, dado que la inclusión en el catálogo responde a razones de buena práctica editorial que, aunque sea lo habitual, no necesariamente va unida a la calidad científica; de igual manera pesó como restricción en la aplicación de estos las inexactitudes detectadas en las declaraciones sobre la práctica del arbitraje de los artículos.

Una vez identificadas las revistas, los criterios de selección de aquellas que serían fuentes se muestran en la tabla 2.

Evidentemente, no todas las revistas puntuaban en las variables seleccionadas, pero en todas ellas se seleccionaron las que estaban en el $50 \%$ mejor puntuado en cada categoría o, considerando la puntuación más alta obtenida, las que alcanzaban al menos un tercio de esta, dependiendo de la naturaleza y características de la variable y de la propia elaboración de los autores originales de estos indicadores.
Tabla 2. Variables tenidas en cuenta en Dialnet Métricas para la selección de las revistas fuente

\begin{tabular}{|c|l|c|l|}
\hline $\mathbf{N}$ & \multicolumn{1}{|c|}{ Variables } & N & \multicolumn{1}{|c|}{ Variables } \\
\hline 1 & Inclusión en WoS & 8 & Cuartil de Scopus \\
\hline 2 & Inclusión en ESCI & 9 & Cuartil en In-RECX \\
\hline 3 & Citas absolutas recibidas en WoS & 10 & h Google \\
\hline 4 & Citas promedio por artículo & 11 & Mediana $h$ Google \\
\hline 5 & CiteScore de Scopus & 12 & Ranking Fecyt \\
\hline 6 & Posición en SJR & 13 & Antigüedad \\
\hline 7 & Cuartil JCR & 14 & Presencia en Dialnet \\
\hline
\end{tabular}

La aplicación de los criterios ha estado marcada por excepciones y particularidades, dado que lo que interesaba era identificar las mejores revistas pero también las que representaban especialidades minoritarias, algunas de Derecho o de Filología. En consecuencia, se incluyeron todas las existentes en WoS-JCR, aunque en líneas generales estas últimas se situaban entre las mejor puntuadas en la mayoría de los restantes criterios.

La selección de revistas fuente en Dialnet Métricas responde por tanto más a una estrategia de complementariedad, pues su objetivo es aportar la información para estudiar las revistas españolas que no se encuentran en las bases de datos de referencias internacionales (Web of Science y Scopus). El proceso es además una vía de acercarse a las que mantienen una calidad aceptable en los indicadores, aunque en el futuro y a medida que el proceso se vaya afinando, el mecanismo de posicionamiento serían las citas recibidas.

En la figura 1 se presenta la página principal de acceso a los componentes principales del índice Dialnet de Revistas (IDR) y los Indicadores Dialnet. Dialnet Métricas incluye indicadores de revistas, investigadores, universidades y áreas de conocimiento y la posibilidad de realizar búsquedas y navegar dentro de todos estos elementos. 


\section{Descripción de Dialnet Métricas}

Dialnet Métricas se estructura en dos áreas:

- una referida a las revistas: Índice Dialnet de Revistas (IDR).

El IDR se calcula a partir de las citas emitidas por los artículos de una selección de revistas, las que se indican en cada edición y aportan datos e indicadores de cada una para los años de edición del índice;

- otra dedicada a autores, universidades, áreas del conocimiento y otras: Indicadores Dialnet.

Éstos se obtienen para cada campo, calculados a partir del conjunto de las referencias contenidas en los artículos incluidos en Dialnet y no sólo de revistas españolas, sino incluso de Latinoamérica y en los documentos publicados en cualquier año (tabla 3).

Tabla 3. Estructura de la web y resumen de apartados de Dialnet Métricas

\begin{tabular}{|c|c|c|}
\hline Sección o apartado & Descripción & Información a la que da acceso \\
\hline Portada (home) & $\begin{array}{l}\text { Acceso a los indicadores de las revistas fuente } \\
\text { resultado del cruce entre Latindex y Dialnet, } \\
\text { así como a indicadores de calidad basados en } \\
\text { citas para otros tipos documentales. }\end{array}$ & Acceso al IDR e Indicadores Dialnet y búsqueda de revistas y autores. \\
\hline $\begin{array}{l}\text { Índice Dialnet de Revistas } \\
(I D R)\end{array}$ & $\begin{array}{l}\text { Muestra las revistas y sus indicadores den- } \\
\text { tro de cada campo. }\end{array}$ & Búsqueda por revista y navegación por cada campo. \\
\hline Indicadores Dialnet & $\begin{array}{l}\text { Aporta indicadores para los distintos campos } \\
\text { temáticos calculados a partir de las citas rea- } \\
\text { lizadas en los artículos que en Dialnet tienen } \\
\text { incluidas las referencias bibliográficas. }\end{array}$ & $\begin{array}{l}\text { Búsqueda por autores permitiendo el acceso al perfil dentro de Dialnet. } \\
\text { La navegación por cada campo brinda acceso a información de los } \\
\text { investigadores más citados, por área de conocimiento y universida- } \\
\text { des, además de las publicaciones más citadas y referencias por año. }\end{array}$ \\
\hline $\begin{array}{l}\text { Información de cada } \\
\text { campo sólo en el IDR }\end{array}$ & $\begin{array}{l}\text { Información general del campo para cada } \\
\text { año evaluado y revistas que lo conforman. }\end{array}$ & $\begin{array}{l}\text { Descripciones del campo, citas emitidas, población de revistas e } \\
\text { índice de coautoría. } \\
\text { Para cada revista se expone el impacto, cantidad de artículo, citas re- } \\
\text { cibidas y el ranking de acuerdo con el índice de impacto y se puede } \\
\text { visualizar la información para cada año evaluado. } \\
\text { Además se accede al listado de revistas del año con la distribución } \\
\text { por campo, así como las citas contabilizadas. }\end{array}$ \\
\hline $\begin{array}{l}\text { Información de cada } \\
\text { revista en el IDR }\end{array}$ & Acceso a indicadores. & $\begin{array}{l}\text { Índice de impacto, citas, posición de la revista y cuartil, triángulo de } \\
\text { citación, además de enlazar con los Indicadores Dialnet, con la distri- } \\
\text { bución de citas recibidas por cada revista por año y la posición de la } \\
\text { revista en el conjunto de todas las de su campo. } \\
\text { Gráficas de tendencia por cuartiles en los distintos años y compara- } \\
\text { ción de distintos indicadores la revista con respecto al total del campo. }\end{array}$ \\
\hline $\begin{array}{l}\text { Investigadores más } \\
\text { citados }\end{array}$ & $\begin{array}{l}\text { Investigadores que aportan al campo por } \\
\text { las distintas áreas y la institución a la que } \\
\text { pertenecen. }\end{array}$ & $\begin{array}{l}\text { Indicadores de producción e impacto, además de enlazar con el } \\
\text { perfil de cada autor en Dialnet de cada universidad y campo. }\end{array}$ \\
\hline $\begin{array}{l}\text { Investigadores por área } \\
\text { de conocimiento }\end{array}$ & $\begin{array}{l}\text { Describe las subcategorías que forman } \\
\text { parte del campo y la información de los } \\
\text { investigadores. }\end{array}$ & $\begin{array}{l}\text { Datos globales del área, como los investigadores, sus indicadores de } \\
\text { producción y citación y la posibilidad de acceder al perfil de cada } \\
\text { investigador y los campos. }\end{array}$ \\
\hline $\begin{array}{l}\text { Investigadores por } \\
\text { universidades }\end{array}$ & $\begin{array}{l}\text { Listado de cada una de las universidades } \\
\text { que registran publicaciones en los distintos } \\
\text { campos. }\end{array}$ & $\begin{array}{l}\text { Información descriptiva e indicadores de los investigadores de cada } \\
\text { universidad. } \\
\text { Áreas para las que aporta información dentro del campo, informa- } \\
\text { ción de la clasificación CIRC } \\
\text { Enlace al perfil de cada universidad }\end{array}$ \\
\hline $\begin{array}{l}\text { Publicaciones más } \\
\text { citadas }\end{array}$ & $\begin{array}{l}\text { Se listan los trabajos más citados del campo } \\
\text { por tipos documentales. }\end{array}$ & $\begin{array}{l}\text { Tipos documentales y años, ranking de trabajos de acuerdo con } \\
\text { distintas variables e indicadores. } \\
\text { Enlaza con el panel del campo al que pertenece la publicación y } \\
\text { con el perfil de esta dentro de Dialnet Métricas y con los indicadores } \\
\text { experimentales. }\end{array}$ \\
\hline $\begin{array}{l}\text { Referencias } \\
\text { bibliográficas por año }\end{array}$ & $\begin{array}{l}\text { Visualiza el grado de vigencia/obsoles- } \\
\text { cencia de la bibliografía científica de } \\
\text { cada campo temático, presentando la } \\
\text { distribución cronológica de las referencias } \\
\text { bibliográficas citadas por los investigadores } \\
\text { en sus publicaciones. }\end{array}$ & $\begin{array}{l}\text { Tanto en gráfico como en tabla, para cada año del índice, se muestra } \\
\text { la anualidad citada con el número de referencias de cada año y el \% } \\
\text { de referencias que representa. } \\
\text { Enlaza con el campo evaluado. }\end{array}$ \\
\hline
\end{tabular}

Figura 1. Página principal de Dialnet Métricas

https://dialnet.unirioja.es/metricas
Índice Dialnet de Revistas e Investigadores O Revistas OInvestigadores

Título de la revista

Indicadores Dialnet Investigadores, universidades, etc. Leer más 


\section{Índice Dialnet de Revistas (IDR)}

Es el instrumento que permite conocer el impacto de las revistas incluidas en cada campo y realizar su evaluación con respecto a las de su especialidad. Pueden conocerse además las métricas para cada campo y revista.

El cálculo de los indicadores de impacto de las revistas se realiza con una ventana de citación de cinco años, que obedece a la necesidad de evaluación de las Ciencias Sociales y Humanidades con comportamientos más lentos en la maduración del proceso de citación y prácticas de producción igualmente distintas a otras áreas y disciplinas. El proceso de citación incluye tanto las citas que reciben y aporta cada revista fuente incluidas en Dialnet, como las procedentes de revistas internacionales (Web of Science) lo que complementa la cobertura internacional de Dialnet siguiendo en este punto la metodología ya empleada en los productos In-RECX.

Tabla 4. Descripción de los indicadores del Índice Dialnet de Revistas (IDR)

\begin{tabular}{|l|l|}
\hline \multicolumn{2}{|c|}{ Indicadores generales por campo, para cada año, como un perfil de cada revista } \\
\hline Población y listado de revistas & \\
\hline Número de publicaciones totales de cada revista & Cantidad de revistas y títulos que forman parte del campo temático. \\
\hline Índice de coautoría & Promedio de autores por artículo en el conjunto de revistas del campo. \\
\hline Citas recibidas & Total de citas de cada revista del campo por año. \\
\hline Índice de impacto IDR & Promedio de citas por documento (C/D) para cada anualidad con una ventana de cinco (5) años. \\
\hline Ranking de revistas & De acuerdo al índice de impacto, se elaboran cuartiles. \\
\hline Artículos citables & $\begin{array}{l}\text { Conteo total y distribución de los artículos citables de la revista dentro de un triángulo de } \\
\text { citación con relación al total de citas del año. }\end{array}$ \\
\hline Posición & Muestra la posición que ocupa dentro de las revistas de la categoría. \\
\hline
\end{tabular}

\section{Indicadores Dialnet}

Se elaboran a partir del contenido completo de la base de datos de Dialnet, a diferencia del IDR en el que se tienen en cuenta sólo las citas emitidas por el conjunto de revistas fuente. En este índice, a partir de las citas recibidas por las publicaciones, se ha trasladado esta información a revistas y autores y desde éstos a su institución y área de conocimiento.

Tabla 5. Descripción de la sección Indicadores Dialnet

\begin{tabular}{|c|c|}
\hline \multicolumn{2}{|r|}{$\begin{array}{l}\text { Investigadores más citados en cada campo. } \\
\text { Se muestran los indicadores de cada investigador, la institución a la que pertenece y el área de trabajo }\end{array}$} \\
\hline Sección & Descripción \\
\hline Número de publicaciones & Cantidad de documentos contabilizados a cada investigador dentro de Dialnet. \\
\hline Citas totales & Número de citas recibidas por investigador que proceden de revistas de Dialnet de todos los campos. \\
\hline Citas dentro de campo & Número total de citas recibidas que proceden de revistas del campo evaluado. \\
\hline Índice $\mathrm{h}$ & $\begin{array}{l}\text { A partir de la distribución de citas de sus trabajos en Dialnet se calcula el índice h, entendido como la cantidad h } \\
\text { de trabajos que han recibido al menos h citas. Se muestra el h5 ( } 5 \text { años) y el promedio del índice de } 10 \text { y } 5 \text { años. }\end{array}$ \\
\hline \multicolumn{2}{|r|}{$\begin{array}{l}\text { Investigadores por área de conocimiento. } \\
\text { Expone el listado e indicadores de los investigadores que aportan producción científica al campo }\end{array}$} \\
\hline Número de publicaciones & Publicaciones existentes en Dialnet en las que ha participado el investigador. \\
\hline Citas & Total de citas recibidas por las publicaciones de cada investigador. \\
\hline Citadas & Publicaciones que han recibido citas, calculadas sobre el total de publicaciones. \\
\hline Número de investigadores & Número total de investigadores que aportan publicaciones al campo. \\
\hline Número de publicaciones & Cantidad de documentos del conjunto de los investigadores de la disciplina. \\
\hline Número de citas & Citas recibidas por el total de publicaciones contabilizadas del campo. \\
\hline Autocitas & Tasa de autocitación o porcentaje de autocitas del campo. \\
\hline Índice $\mathrm{h}$ & $\begin{array}{l}\text { A partir de la distribución de citas de sus trabajos en Dialnet, entendido como la cantidad de trabajos h que han } \\
\text { recibido al menos h citas. Se muestra el h5 ( } 5 \text { años) y el promedio del índice de } 10 \text { y } 5 \text { años. }\end{array}$ \\
\hline \multicolumn{2}{|r|}{$\begin{array}{l}\text { Investigadores por universidades. } \\
\text { Se calculan indicadores para las universidades que aportan investigación a cada campo }\end{array}$} \\
\hline Número de investigadores & Número total de investigadores de las universidades que trabajan en el campo. \\
\hline Número de publicaciones & Cantidad de publicaciones de la universidad. \\
\hline Citas & Citas recibidas por la universidad distribuidas por las distintas subdisciplinas en las que trabaja. \\
\hline Autocitas & Tasa de autocitación de la universidad. \\
\hline Índice h & $\begin{array}{l}\text { A partir de la distribución de citas de sus trabajos en Dialnet se expone el índice h, el h5 (5 años) y el promedio } \\
\text { del índice de } 10 \text { y } 5 \text { años. }\end{array}$ \\
\hline
\end{tabular}




\begin{tabular}{|l|l|}
\hline \multicolumn{3}{|c|}{$\begin{array}{c}\text { Listado de los investigadores de la universidad y el área en la que trabajan } \\
\text { Desde el área de cada investigador enlaza con el listado de investigadores por área y desde el nombre del investigador con el perfil de cada } \\
\text { uno en Dialnet }\end{array}$} \\
\hline Número de publicaciones & Número de publicaciones que hay en Dialnet en las que ha participado el investigador. \\
\hline Citas recibidas & Total de citas que han recibido sus publicaciones. \\
\hline Citadas & Cantidad de publicaciones que han recibido citas. \\
\hline Sin citar & Cantidad de publicaciones que no han recibido citas. \\
\hline \multicolumn{3}{|l|}{$\begin{array}{l}\text { Indicadores de publicaciones. } \\
\text { Citas totales }\end{array}$} & Citas totales que provienen de revistas de los campos cubiertos por Dialnet. \\
\hline Citas dentro de campo & Citas totales que provienen de revistas del campo evaluado. \\
\hline \multicolumn{2}{|r|}{$\begin{array}{r}\text { Referencias bibliográficas } \\
\text { Se visualiza el grado de vigencia/obsolescencia de la bibliografía científica del campo, a partir de la distribución cronológica de las referencias } \\
\text { bibliográficas citadas por los investigadores en sus publicaciones. }\end{array}$} \\
\hline
\end{tabular}

\section{Volumen de datos de Dialnet Métricas}

La descripción del volumen de datos de Dialnet Métricas tiene como objetivo mostrar y comentar los datos principales del tamaño de la base. Aunque continuará aumentando a medida que se incluyan años y nuevos campos temáticos, aporta una perspectiva general de la información contenida en la misma utilizada para la obtención de los indicadores y la forma que puede crecer en el tiempo.

Se exponen tanto los datos relacionados con el total de referencias empleadas y citas emitidas, como la cantidad de revistas fuente de todos los campos en cada año. En las tablas 6 y 7 se puede seguir el incremento de las cifras de citas emitidas, unas 2.300 en promedio ( $10 \%$ anual). Se observa además la disminución del número de revistas fuente, el aumento del volumen de citas emitidas, la cantidad de revistas fuente en cada año de elaboración del índice con respecto a la población total de las relacionadas con el campo.

Con respecto a la selección de revistas, actualmente se han incorporado revistas de 40 campos segmentados en 12 de Ciencias Sociales, 16 de Humanidades y 12 en Derecho, para una población de 2.472 , de las cuales se ha empleado algo más del $25 \%$. Esta selección obedece a la distribución tipo Bradford que se da en las revistas científicas a la hora de concentrar la información, según la cual la selección de un grupo reducido de revistas permite identificar la mayor cantidad de información y la más representativa y, en esa misma medida, a las revistas más relevantes de cada disciplina.

Tabla 7. Análisis de las revistas que forman parte de los campos temáticos de Dialnet Métricas (abril 2021)

\begin{tabular}{|c|c|c|c|c|c|}
\hline Campos temáticos & Revistas totales & Revistas fuente & \% de selección & $\begin{array}{c}\text { Fuentes en más de } \\
\text { un campo }\end{array}$ & $\begin{array}{c}\text { Solapamiento fuen- } \\
\text { tes (\%) }\end{array}$ \\
\hline Antropología & 25 & 10 & 40,00 & 6 & 60,00 \\
\hline Bellas artes & 138 & 29 & 21,01 & 6 & 20,69 \\
\hline Ciencias Políticas & 90 & 24 & 26,67 & 14 & 58,33 \\
\hline Comunicación & 61 & 18 & 29,51 & 7 & 38,89 \\
\hline Deporte & 46 & 24 & 52,17 & 3 & 12,50 \\
\hline Derecho & 358 & 100 & 27,93 & 26 & 26,00 \\
\hline Documentación & 23 & 16 & 69,57 & 2 & 12,50 \\
\hline Economía & 174 & 44 & 25,29 & 13 & 29,55 \\
\hline Educación & 235 & 59 & 25,11 & 8 & 13,56 \\
\hline Estudios de género & 24 & 12 & 50,00 & 1 & 8,33 \\
\hline América Latina & 29 & 7 & 24,14 & 7 & 100,00 \\
\hline Filologías & 328 & 84 & 25,61 & 17 & 20,24 \\
\hline Filosofía & 100 & 25 & 25,00 & 10 & 40,00 \\
\hline Geografía & 43 & 14 & 32,56 & 3 & 21,43 \\
\hline Historia & 319 & 84 & 26,33 & 30 & 35,71 \\
\hline Multidisciplinares & 142 & 25 & 17,61 & 12 & 48,00 \\
\hline Psicología & 121 & 30 & 24,79 & 8 & 26,67 \\
\hline Religión & 67 & 17 & 25,37 & 4 & 23,53 \\
\hline Sociología & 92 & 23 & 25,00 & 9 & 39,13 \\
\hline Trabajo social & 57 & 13 & 22,81 & 5 & 38,46 \\
\hline Total & 2.472 & 658 & 26,62 & - & 13,53 \\
\hline
\end{tabular}

* La tasa de solapamiento muestra la presencia de revistas en más de un campo. Una revista puede estar en dos o más, por ello los acumulados por campos pueden no coincidir con los parciales. 
El número de revistas españolas incluidas en Dialnet es 3 veces mayor que en $S J R$ y 20 veces mayor que en $J C R$ (tabla 8). Esto no significa que la base sea mejor, pero sí que es claramente más exhaustiva. La información e indicadores por áreas en campos cuya internacionalización es todavía precaria, resulta una de las aportaciones más importantes de DM, permitiendo a investigadores y evaluadores contar con información clave de apoyo a sus decisiones.

\section{Visualización de información en Dialnet Métricas}

Elementos importantes de Dialnet Métricas son los gráficos que facilita, tanto de tendencia como de barras, que garantizan la mejor comprensión de la información resultante, tanto dentro del IDR como en los Indicadores Dialnet y de los cuales se proporcionan ejemplos. En el apartado de revistas dentro del IDR se presenta un triángulo de citación para cada revista, que exhibe para los años de publicación la cantidad de artículos citables y la evolución de las citas recibidas por una determinada revista en un período de tiempo, y de manera específica la distribución de citas por año, la sumatoria tanto por año de publicación como en el que se reciben las citas y el IDR en cada año (figura 2).

Dialnet Métricas provee gráficos que muestran las citas recibidas por tipos documentales y de acuerdo a la Clasificación Integrada de Revistas Científicas (CIRC) para los autores, y esta última también para las instituciones y artículos (figura 3), además se observa en forma de tendencia la distribución de las citas recibidas tanto por año de emisión como de publicación (figura 4). Para cada campo se expone la vigencia/obsolescencia de la bibliografía generada (figura 5), es decir, en los años citantes podemos conocer la cantidad de referencias utilizadas que pertenecen a años anteriores (año citado), y de esta forma es posible conocer las tendencias de los campos con relación a la actualidad de la información que emplea para la generación de nuevo conocimiento.

Triángulo de citación

\begin{tabular}{|c|c|c|c|c|c|}
\hline Años & $\begin{array}{r}\text { Artículos } \\
\text { citables }\end{array}$ & 2016 & 2017 & 2018 & 2019 \\
\hline 2011 & 12 & 12 & - & - & - \\
\hline 2012 & 12 & 4 & 口 & - & - \\
\hline 2013 & 12 & $\square$ & $\square$ & $\square$ & - \\
\hline 2014 & 12 & I & $\|$ & 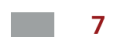 & 12 \\
\hline 2015 & 14 & I & 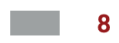 & 【 & $\|$ \\
\hline 2016 & 16 & - & $\square$ & 18 & 35 \\
\hline 2017 & 15 & - & - & $\square$ & 6 \\
\hline 2018 & 19 & - & - & - & $\square$ \\
\hline Total de artículos & 112 & & & & \\
\hline Citas por año & & 22 & 31 & 35 & 65 \\
\hline $\begin{array}{l}\text { Índice de impacto } \\
\text { IDR }\end{array}$ & & 0,355 & 0,470 & 0,507 & 0,855 \\
\hline
\end{tabular}

Figura 2. Triángulo de citación de una revista

\begin{tabular}{|c|c|c|c|}
\hline Campos temáticos & $\begin{array}{l}\text { Dialnet } \\
\text { Métricas }\end{array}$ & SJR 2019 & JCR 2019 \\
\hline Antropología & 25 & 11 & 2 \\
\hline Bellas artes & 138 & 131 & 9 \\
\hline Ciencias políticas & 90 & 15 & 2 \\
\hline Comunicación & 61 & 21 & 3 \\
\hline Deporte & 46 & 9 & 1 \\
\hline Derecho & 358 & 26 & 3 \\
\hline Documentación & 23 & 8 & 2 \\
\hline Economía & 174 & 24 & 9 \\
\hline Educación & 235 & 60 & 9 \\
\hline Estudios de género & 24 & 4 & 0 \\
\hline América Latina & 29 & - & - \\
\hline Filologías & 328 & 180 & 21 \\
\hline Filosofía & 100 & 36 & 11 \\
\hline Geografía & 43 & 21 & 2 \\
\hline Historia & 319 & 98 & 20 \\
\hline Multidisciplinares & 142 & 40 & 11 \\
\hline Psicología & 121 & 40 & 14 \\
\hline Religión & 67 & 17 & 0 \\
\hline Sociología & 92 & 41 & 5 \\
\hline Trabajo social & 57 & 1 & 0 \\
\hline
\end{tabular}

Notas:

Sólo se tienen en cuenta las revistas Core de Web of Science sin ESCI.

Los datos del $S J R$ y $J C R$ pueden ser aproximados, dado que algunas categorías presentan denominaciones diferentes o existen duplicados de revistas por lo que es difícil un alto nivel de precisión.

Tabla 8. Comparativa de la cantidad de revistas españolas por campo en Dialnet Métricas, SCImago Journal Rank y Journal Citation Reports 2019 

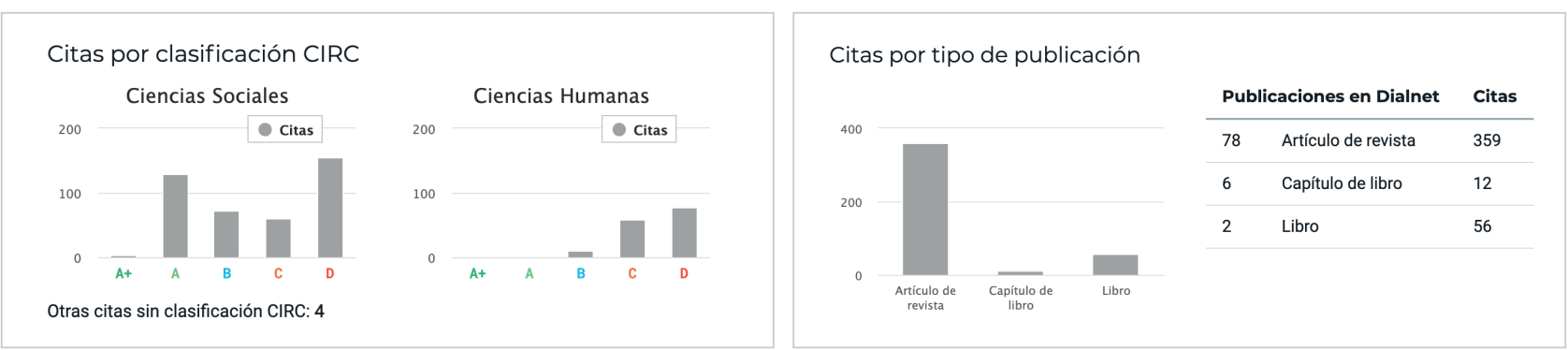

Figura 3. Citas por tipo de publicación y de acuerdo a la CIRC

Citas por año de emisión

Gráfico Tabla

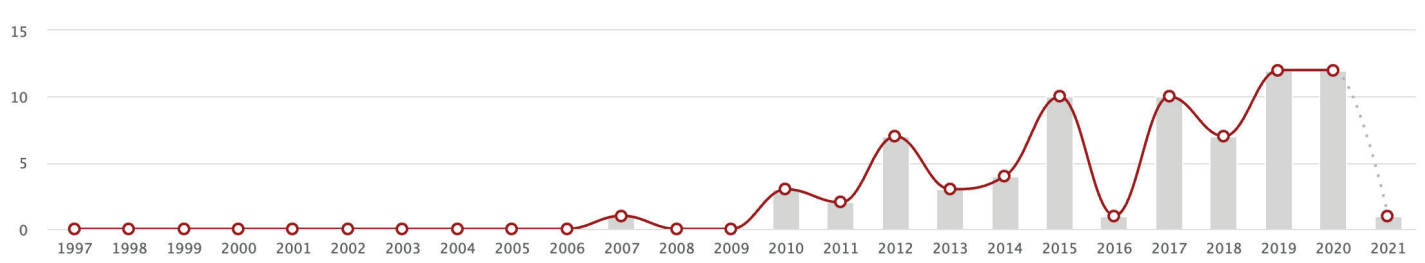

Citas por año de publicación

Gráfico Tabla

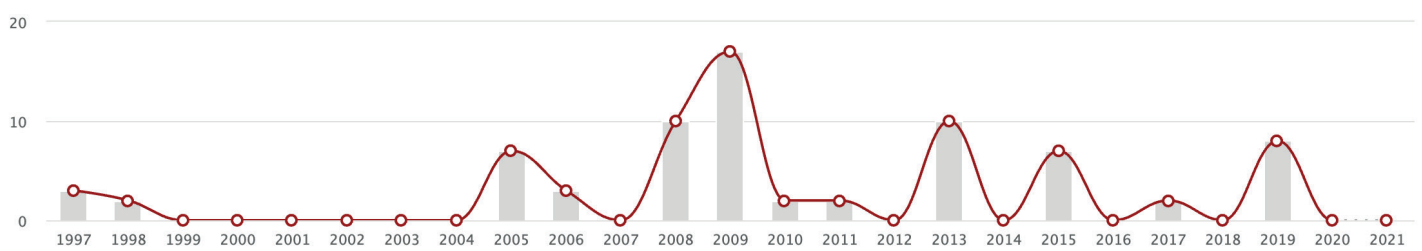

Figura 4. Tendencia de las citas por año de emisión y de publicación en Dialnet Métricas

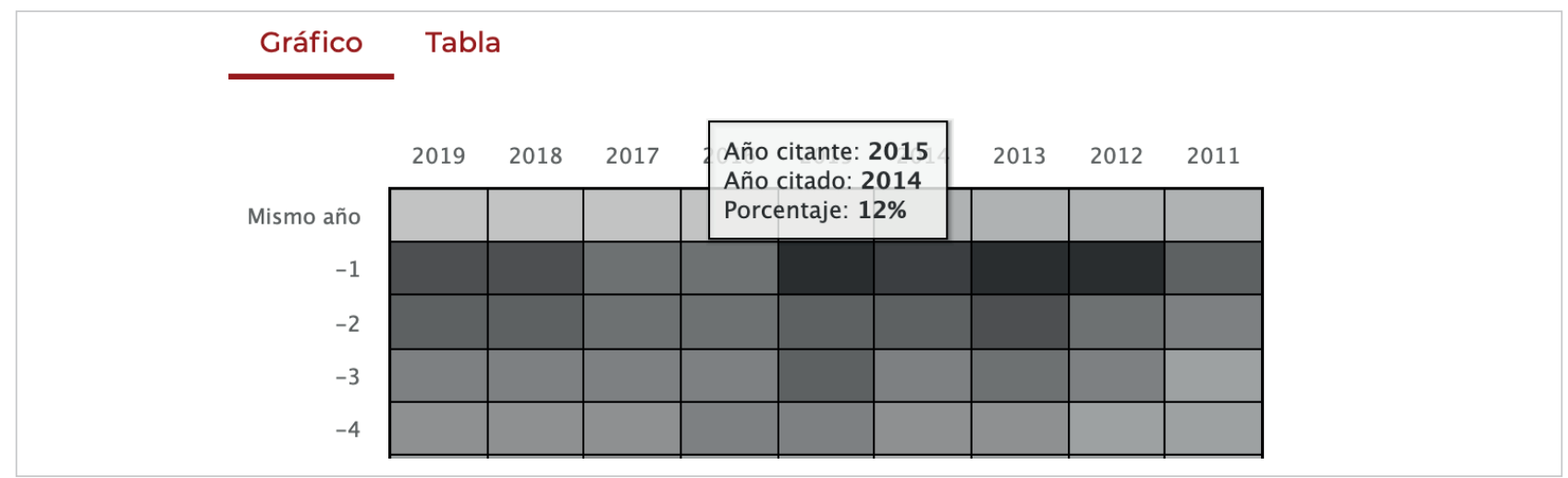

Figura 5. Vigencia/obsolescencia de la bibliografía científica en un campo de Dialnet Métricas

\section{Discusión y uso de Dialnet Métricas}

Dialnet Métricas (DM) llega y se consolida en una etapa clave para cubrir y ayudar a solventar la problemática de la obtención de indicadores y la evaluación de las Ciencias Sociales y las Humanidades y especialmente para estudiar los circuitos nacionales de comunicación científica, puesto que los internacionales ya están sobrecubiertos con un surtido grupo de productos.

Como índice de evaluación es una iniciativa transparente que creemos aporta información significativa para la toma de decisiones en la investigación y evaluación científica, dado que los indicadores bibliométricos favorecen el análisis del comportamiento, no sólo de revistas, sino
Debido a la insuficiencia de los recursos disponibles, por abandono de algunos y por falta de información bibliométrica de otros, surge Dialnet Métricas 
de investigadores y de otros actores del proceso científico como instituciones o áreas. Todo este conjunto de recursos será útil para las agencias de evaluación, responsables editoriales y científicos que podrán tomar decisiones basadas en un número de elementos mucho mayor y más variado, algunos de los cuales sólo son accesibles a través de esta plataforma.
La elaboración del índice partió de la identificación de las poblaciones de revistas de cada especialidad y la selección de la muestra de revistas con mayor representatividad

El hecho de que en las Ciencias Sociales y Humanidades exista la tendencia a publicar una cantidad significativa de resultados de investigación en revistas nacionales, y a citar también sus revistas, le añade un valor estratégico al índice, que como se mencionó, ayuda a la evaluación de las disciplinas con menor reflejo de los índices internacionales de citación.

Como proyecto colaborativo, merece destacarse la participación de un amplio número de bibliotecas de universidades españolas que en la actualidad cooperan con el proyecto, labor importante dados los esfuerzos que requiere el proceso de gestión de citas, limpieza de literales y validación de resultados de un trabajo de tal magnitud y que le otorga al proceso una garantía adicional de continuidad en el tiempo. A todo esto, una de las tareas más interesantes del proyecto es la coordinación de sus elementos productivos.

Dialnet Métricas se muestra como el sucesor del proyecto In-recs, y destaca sobre el resto de otros que se encargan de la evaluación de revistas por su exhaustiva cobertura en cuanto a revistas españolas, su gratuidad y poseer datos propios (independencia frente a otros productos), por trabajar a niveles inferiores al agregado de revista (artículo, autor), y la contextualización de las métricas por áreas. Los datos de Dialnet Métricas superan con creces a los de otros proyectos españoles de evaluación de revistas.

A la fecha, y con la última ampliación (abril 21), están representadas todas las Ciencias Sociales, las Humanidades y el Derecho, con la posibilidad de conocer detalles de las subespecialidades de algunos campos como Historia y Filología, lo que mejorará sin duda el índice con la posibilidad no sólo de analizar un conjunto mayor de revistas, sino de contar con indicadores para evaluar la actividad científica.

Su futuro debe tener en cuenta el aumento de la cobertura de revistas y sobre todo incorporar a las latinoamericanas del área creando un espacio común con España en las áreas de Ciencias Sociales y Humanidades. Sin embargo, también se tendrán que tomar decisiones difíciles, como qué hacer con las prácticas fraudulentas y aquellos que las realizan, aunque creemos que hoy, en las Ciencias Sociales y Humanidades en España, este es un fenómeno marginal.

Asimismo, el aumento del número de revistas y los años de indización de las referencias bibliográficas, requiere de una rigurosa labor en la que se espera que las bibliotecas que elaboran el sistema realicen un trabajo retrospectivo hasta el año 2010. Se prevé una ampliación de la indización de referencias bibliográficas más allá de la actual (artículos de revistas), incorporando las referencias de los capítulos de libros, los libros o las tesis doctorales.

Por supuesto, los avances de Dialnet Métricas van ligados a Dialnet, dado que conforman un mismo sistema de información y en la medida que se pueda extender el marco de colaboración bibliotecaria en América Latina se podrá disponer de un sistema de información integral, que englobe el conjunto de publicaciones (revistas, libros y capítulos de libros colectivos) del ámbito iberoamericano. A partir de este resultado, se podría disponer de un sistema utilizado en los procesos de evaluación de los investigadores de Ciencias Sociales, Derecho y Humanidades de todos los países de este espacio geográfico.

Habrá un crecimiento de los indicadores, en la medida que en Dialnet se disponga de un elevado porcentaje de los libros académicos publicados en España (más adelante debiera ocurrir lo mismo con los de Iberoamérica), que permitirá evaluar el impacto de las editoriales de libros y cada una de las colecciones de estas editoriales, a partir de las citas que reciben. Del mismo modo se mejorará la cobertura estableciendo apartados para conocer las citas recibidas por las publicaciones y resultados de investigación en las que los investigadores tienen roles distintos, por ejemplo traductores de una publicación, editores literarios de una obra clásica, coordinadores de un libro colectivo, directores de una tesis doctoral.

Finalmente, es necesario insistir en la importancia de la ampliación de la colaboración bibliotecaria, sin la cual no se podría llegar a alcanzar metas ambiciosas. Para Dialnet Métricas como proyecto colaborativo, las bibliotecas universitarias cumplen un rol de liderazgo no sólo para la inclusión y análisis de información, sino como puente con las instituciones y la comunidad científica, de forma tal que pueda afrontarse tanto el escalamiento del sistema como su continuidad con las mayores garantías. 


\section{Referencias}

Abejón-Peña, Teresa (2018). "Presentación de Redib, Red Iberoamericana de Innovación y Conocimiento Científico". Revista española de documentación científica, v. 41, n. 1, e200.

http://redc.revistas.csic.es/index.php/redc/article/view/1004

Aguado-López, Eduardo; Rogel-Salazar, Rosario; Garduño-Oropeza, Gustavo; Zúñiga, María-Fernanda (2008). “Re$d A L y C$ : una alternativa a las asimetrías en la distribución del conocimiento científico". Ciencia, docencia y tecnología, v. 19, n. 37, pp. 11-30.

http://www.revistacdyt.uner.edu.ar/spanish/cdt_37/documentos/37_aguado.pdf

Alperin, Juan-Pablo; Rozemblum, Cecilia (2017). "La reinterpretación de visibilidad y calidad en las nuevas políticas de evaluación de revistas científicas". Revista interamericana de bibliotecología, v. 40, n. 3, pp. 231-241.

https://doi.org/10.17533/udea.rib.v40n3a04

Charum, Jorge (2004). "La construcción de un sistema nacional de indexación, el caso de Publindex". Convergencia. Revista de ciencias sociales, v. 11, n. 35, pp. 293-309.

https://www.redalyc.org/pdf/105/10503511.pdf

Chavarro, Diego; Ràfols, Ismael; Tang, Puay (2018). "To what extent is inclusion in the Web of Science an indicator of journal 'quality'?". Research evaluation, v. 27, n. 2, pp. 106-118.

https://doi.org/10.1093/reseval/rvy001

Córdoba-González, Saray; Murillo-Goussen, Guillermo; Polanco-Cortés, Jorge (2017). "Génesis y desarrollo de UCR index en la Universidad de Costa Rica". E-ciencias de la información, v. 7, n. 1, pp. 54-77.

https://doi.org/10.15517/eci.v7i1.25713

Delgado-López-Cózar, Emilio (2017). “Evaluar revistas científicas: un afán con mucho presente y pasado e incierto futuro". En: Abadal, Ernest (ed.). Revistas científicas: situación actual y retos de futuro. Universitat de Barcelona, pp. 73-103. ISBN: 9788491680383

Delgado-López-Cózar, Emilio; Jiménez-Contreras, Evaristo; Ruiz-Pérez, Rafael; López-Herrera, Antonio-Gabriel; Gacto-Colorado, María-José; Torres-Salinas, Daniel; De-la-Moneda-Corrochano, Mercedes; Ruiz-Baños, Rosario; Pérez-Ortega, Juan-Manuel; Bailón-Moreno, Rafael; Poyatos-Huertas, Encarnación; Rodríguez-Gálvez, María-José (2005). “Inrecs: índice de impacto de las revistas españolas de ciencias sociales". Biblio 3W, Revista bibliográfica de Geografía y Ciencias Sociales, v. 10, n. 574.

http://www.ub.edu/geocrit/b3w-574.htm

Dialnet (2020). Dialnet Métricas.

https://dialnet.unirioja.es/metricas

Garfield, Eugene (1955). "Citation indexes for science: A new dimension in Documentation through association of ideas". Science, v. 122, n. 3159, pp. 108-111.

https://doi.org/10.1126/science.122.3159.108

Garfield, Eugene (1999). “Journal impact factor: a brief review". Canadian Medical Association journal, v. 161, n. 8, pp. 979-80.

https://www.ncbi.nlm.nih.gov/pmc/articles/PMC1230709/pdf/cmaj_161_8_979.pdf

Garfield, Eugene (2003). "The meaning of the impact factor". International journal of clinical and health psychology, v. 3, n. 2, pp. 363-369.

http://www.aepc.es/ijchp/articulos_pdf/ijchp-77.pdf

Giménez-Toledo, Elea (2015). “La evaluación de la producción científica: breve análisis crítico”. Relieve. Revista electrónica de investigación y evaluación educativa, v. 2, n. 1.

https://doi.org/10.7203/relieve.21.1.5160

Giménez-Toledo, Elea (2018). "La evaluación de las Humanidades y de las Ciencias Sociales en revisión”. Revista española de documentacion cientifica, v. 41, n. 3, e208.

https://doi.org/10.3989/redc.2018.3.1552

López-López, Wilson (2018). “Sobre la evaluación de la investigación y los investigadores: Criticas a las métricas y recomendaciones". Universitas psychologica, v. 17, n. 4.

https://doi.org/10.11144/Javeriana.upsy17-4.seii

Martínez-Ávila, Daniel (2019). “Qualis periódicos: el sistema brasileño de evaluación de revistas”. Anuario ThinkEPI, v. 13, e13e01.

https://doi.org/10.3145/thinkepi.2019.e13e01 
Packer, Abel-Laerte; Prat, Anna-Maria; Luccisano, Adriana; Montanari, Fabiana; Santos, Solange; Meneghini, Rogério (2006). "El modelo SciELO de publicación científica de calidad en acceso abierto". En: Babini, Dominique; Fraga, Jorge (eds.). Edición electrónica, bibliotecas virtuales y portales para las ciencias sociales en América Latina y El Caribe. Buenos Aires: Consejo Latinoamericano de Ciencias Sociales, pp. 191-208. ISBN: 9871183534 http://eprints.rclis.org/8270/1/Babinientero.pdf

Rodríguez-Gairín, Josep-Manuel; Somoza-Fernández, Marta; Urbano, Cristóbal (2011). “MIAR: hacia un entorno colaborativo de editores, autores y evaluadores de revistas". El profesional de la información, v. 20, n. 5, pp. 589-595. https://doi.org/10.3145/epi.2011.sep.15

Torres-Salinas, Daniel; Bordons, María; Giménez-Toledo, Elea; Delgado-López-Cózar, Emilio; Jiménez-Contreras, Evaristo; Sanz-Casado, Elías (2010). "Clasificación integrada de revistas científicas (CIRC): propuesta de categorización de las revistas de ciencias sociales y humanas". El profesional de la información, v. 19, n. 6, pp. 675-683.

https://doi.org/10.3145/epi.2010.nov.15

Torres-Salinas, Daniel; Jiménez-Contreras, Evaristo (2010). "Introducción y estudio comparativo de los nuevos indicadores de citación sobre revistas científicas en Journal Citation Reports y Scopus". El profesional de la información, v. 19, n. 2, pp. 201-207.

https://doi.org/10.3145/epi.2010.mar.12

Torres-Salinas, Daniel; Repiso, Rafael (2016). “Clasificación CIRC 2016”. Anuario ThinkEPI, v. 10, pp. $237-239$. https://doi.org/10.3145/thinkepi.2016.47

Vasen, Federico; Lujano-Vilchis, Ivonne (2017). "Sistemas nacionales de clasificación de revistas científicas en América Latina: tendencias recientes e implicaciones para la evaluación académica en ciencias sociales". Revista mexicana de ciencias políticas y sociales, v. 62, n. 231, pp. 199-228.

https://doi.org/10.1016/S0185-1918(17)30043-0

\section{Anexos}

Anexo 1. Datos generales sobre Dialnet Métricas (abril de 2021)

\begin{tabular}{|l|l|r|}
\hline \multicolumn{2}{|l|}{ Artículos con referencias } & 392.701 \\
\hline Número de referencias & 12.021 .635 \\
\hline Referencias que citan documentos que están en Dialnet & 5.060 .479 \\
\hline \multirow{4}{*}{ Tipos de documentos citados } & artículos de revista & 2.862 .727 \\
\cline { 2 - 3 } & libros & 1.569 .837 \\
\cline { 2 - 3 } & capítulos de libros colectivos & 513.462 \\
\cline { 2 - 3 } & tesis doctorales & 114.453 \\
\hline
\end{tabular}

\section{Anexo 2. Datos de las revistas fuente (26 de abril de 2021)}

\begin{tabular}{|l|l|c|}
\hline \multicolumn{2}{|l|}{ Artículos con referencias } & 139.906 \\
\hline Número de referencias & 4.909 .979 \\
\hline \multicolumn{2}{|l|}{ Referencias que citan documentos que están en Dialnet } & 2.806 .153 \\
\hline \multirow{4}{*}{ Tipos de documentos citados } & artículos de revista & 1.510 .481 \\
\cline { 2 - 4 } & libros & 892.208 \\
\cline { 2 - 3 } & capítulos de libros colectivos & 338.385 \\
\cline { 2 - 3 } & tesis doctorales & 65.079 \\
\hline
\end{tabular}




\section{Desde la investigación en psicología hasta la práctica clínica}

\section{Descubra los recursos confiables de APA}

\section{Índice esencial}

\section{APA PsycInfo ${ }^{\circledR}$}

El índice de psicología más completo del mundo, que contiene millones de registros bibliográficos y se remonta al siglo XVII

\section{Herramientas de prueba}

\section{APA PsycBooks ${ }^{\circledR}$}

Textos completos de capítulos de libros académicos y profesionales publicados por la APA, incluyendo obras de la serie

APA Handbooks in Pschology ${ }^{\circledR}$.

\section{APA PsycArticles ${ }^{\circledR}$}

Artículos a texto completo revisados por pares, publicados por APA y revistas asociadas

\section{APA PsycTests ${ }^{\circledR}$} investigación
Miles de pruebas psicológicas, escalas e instrumentos para la

\section{Literatura gris}

\section{APA PsycExtra ${ }^{\circledR}$}

Fuentes difíciles de encontrar que no están incluidas en APA PsycInfo, como actas de conferencias, informes, normas y directrices, etc.

\section{Vídeos de terapias}

\section{APA PsycTherapy ${ }^{\circledR}$}

Cientos de vídeos sin guiones que muestran a los profesionales clínicos demostrando técnicas de terapia con fines de formación

Junto con APA PsycInfo ${ }^{\circledR}$ (la principal base de datos de resúmenes e indización),

la American Psychological Association (APA) publica recursos bibliográficos, de texto completo y multimedia, todos indizados utilizando el Thesaurus of Psychological Index Terms ${ }^{\circledR}$ de APA.

\section{Para activar una prueba gratuita, póngase en contacto con su representante de EBSCO o visite: trustapa.is/14}

